\title{
The High Energy Particle Detector onboard CSES-02 satellite
}

\author{
Valentina Scotti* \\ Università degli Studi di Napoli Federico II, Dipartimento di Fisica, Napoli, Italy and Istituto \\ Nazionale di Fisica Nucleare - Sezione di Napoli, Italy \\ E-mail: scottiv@na.infn.it
}

\section{Giuseppe Osteria}

Istituto Nazionale di Fisica Nucleare - Sezione di Napoli, Italy

\section{for the CSES-Limadou Collaboration ${ }^{\dagger}$}

\begin{abstract}
In this paper we will present a description of the High Energy Particle Detector (HEPD-02) developed for the China Seismo Electromagnetic Satellite (CSES) project.

CSES is a scientific mission dedicated to monitoring electromagnetic, plasma and particles perturbations of atmosphere and inner Van Allen belts caused by solar and terrestrial phenomena, and to the study of the low energy component of the cosmic rays ( $3-100 \mathrm{MeV}$ for electrons and 30 - $200 \mathrm{MeV}$ for protons).

The first satellite, launched in 2018, hosted several instruments onboard. The HEPD-01, built by the Italian "Limadou" collaboration, is the instrument devoted to separate electrons and protons, as well as light nuclei in the $\mathrm{MeV}$ energy window. The detector is composed of two planes of double-sided silicon microstrip detectors which give the direction of the incident particle, a segmented plastic scintillator for trigger and a calorimeter.

The CSES-02 satellite is planned to be launched by the end of 2021. The next generation HEPD foresees improvements both on the tracker and the calorimeter. To improve trigger efficiency a new system with crossed layers of $2 \mathrm{~mm}$ thick segmented counters read by light-guides has been designed. A new tracker study is ongoing: 3 planes of ALPIDE CMOS pixel chips, developed for ALICE experiment at LHC.
\end{abstract}

36th International Cosmic Ray Conference -ICRC2019-

July 24th - August 1st, 2019

Madison, WI, U.S.A.

\footnotetext{
* Speaker.

${ }^{\dagger}$ for collaboration list see PoS(ICRC2019)1177
} 


\section{Introduction}

CSES (China Seismo-Electromagnetic Satellite) is a scientific mission dedicated to monitoring electromagnetic, plasma and particles perturbations of the atmosphere, ionosphere, magnetosphere and Van Allen belts induced by natural sources and anthropocentric emitters and to study their correlations with the occurrence of seismic events.

The CSES mission is part of a collaboration program between the China National Space Administration (CNSA) and the Italian Space Agency (ASI). The project is developed by China Earthquake Administration (CEA) and Italian National Institute for Nuclear Physics (INFN), together with several Chinese and Italian Universities and research Institutes.

CSES program aims at building a space monitoring system to investigate the topside ionosphere with the most advanced techniques and equipment, and designed in order to gather worldwide data of the near-Earth electromagnetic environment. The observations will provide an observations sharing service for international cooperation and the scientific community.

CSES program foresees the realization of a constellation of satellites. The first satellite of the constellation, CSES-01, was launched in 2018, and inserted into a circular Sun-syncronous orbit with $98^{\circ}$ inclination and $500 \mathrm{~km}$ altitude. In order to perform a comprehensive study of ionospheric phenomena, the mission takes advantage of a multi-instrument payload comprising nine detectors. All the payloads are working at latitude comprised between $\pm 65^{\circ}$.

One of the instruments on-board is the High Energy Particle Detector (HEPD-01), built by the Italian "Limadou" Collaboration. The main task of this high-precision instrument is to detect electrons in the energy range between 3 and $100 \mathrm{MeV}$, and protons between 30 and $200 \mathrm{MeV}$, as well as light nuclei in the $\mathrm{MeV}$ energy window.

The CSES-02 satellite is planned to be launched by the end of 2021. It will be placed on the same orbital plane of CSES-01, with a phase shift of $180^{\circ}$ with respect to the first satellite, in order to optimize the timing between two passages over the same site, and to reduce the temporal resolution. Fig. 1 shows the simulated tracking of the orbit of the two satellites.

All the instruments onboard CSES-02 will work along the whole orbit. Every payload will collect data in two operating modes: "burst mode" and "survey mode". The burst mode is activated when the satellite passes over the whole China territory and the regions with the strongest seismic activities in the world, while over all the other areas, the survey mode will be used. The next generation HEPD, built upon the experience of HEPD-01, foresees improvements to some parts of the system, with substantial changes on part of the apparatus, which will be described in the following.

\section{HEPD-02}

The High Energy Particle Detector will study low energy Cosmic Rays in the energy range 3 $300 \mathrm{MeV}$. For each particle, the HEPD instruments can identify the type (proton, electron, nucleus), measure the energy and the pitch angle. HEPD-02 can therefore detect flows of particles coming from the Van Allen belts and determine the magnetospheric region of origin with great accuracy. The high-inclination orbit allows the HEPD-02 to detect particles of different nature during its revolution: galactic cosmic rays, particles trapped in the magnetosphere, and also solar energetic 


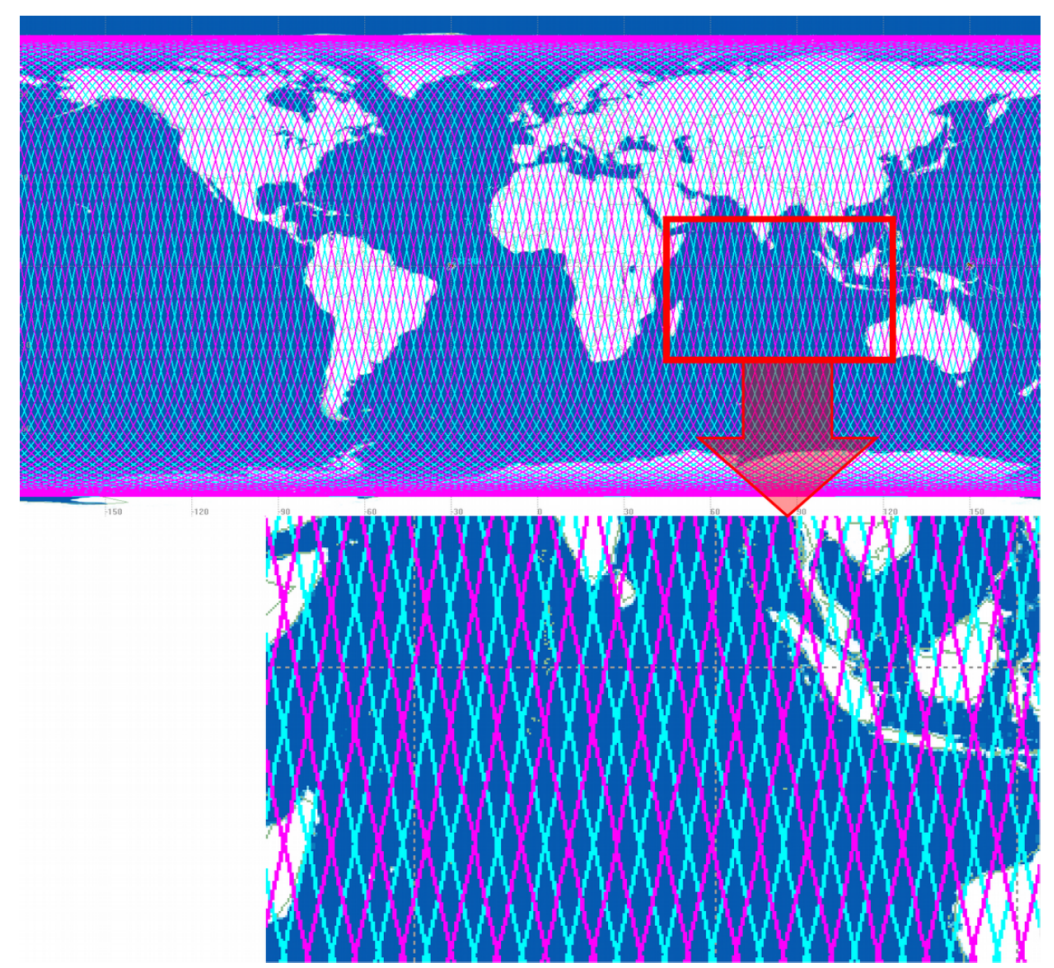

Figure 1: Simulation of the orbital tracking of CSES-01 and CSES-02. The figure shows the $180^{\circ}$ phase shift between the two orbits.

particles associated with transient phenomena such as Solar Flares or Coronal Mass Ejections. To achieve the best result it is necessary to maximize the geometrical acceptance according to weight and power budgets constraints. Some of the main parameters of HEPD-02 are summarized in table 1.

\begin{tabular}{cc}
\hline Parameter & Value \\
\hline Energy Range & Electrons: $3-100 \mathrm{MeV}$ \\
Protons: $30-200 \mathrm{MeV}$ \\
Angular resolution & Electrons: $<10^{\circ}$ with $\mathrm{E}>3 \mathrm{MeV}$ \\
Energy resolution & Electrons: $<10 \%$ with E $>5 \mathrm{MeV}$ \\
Pointing & Zenith \\
Operative temperature & $-10^{\circ}+35^{\circ}$ \\
Mass & $<45 \mathrm{~kg}$ \\
Power Consumption & $<45 \mathrm{~W}$ \\
Data budget & $<100 \mathrm{~Gb} /$ day \\
Life cycle & $>6$ years \\
\hline
\end{tabular}

Table 1: HEPD-02 main technical characteristics.

The HEPD is composed of several detectors (see Fig. 2). A tracker is located on the top of the instrument in order to limit the effect of Coulomb multiple scattering on the direction measurement. Just below, a plane made of two crossed layers of plastic scintillators gives the trigger; this plane is 


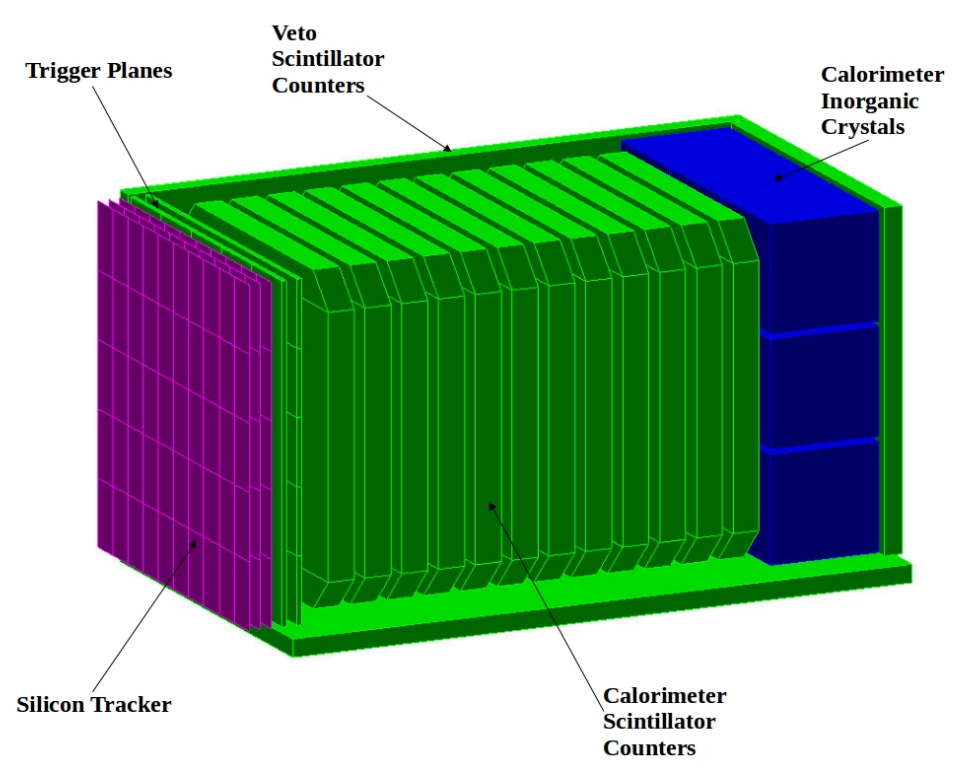

Figure 2: The architecture of the HEPD-02 onboard CSES-02 satellite.

followed by a calorimeter, constituted by a tower of plastic scintillators, followed by a $3 \times 3$ matrix of an inorganic scintillator LYSO. The calorimeter volume is surrounded by plastic scintillator veto planes.

As was previously mentioned, the HEPD-02 onboard CSES-02 will be operational along all the orbit, this situation sets additional and more stringent demands on the design:

- the high trigger rate at polar regions requires an improved trigger logic and the necessity of pre-scaling;

- the larger amount of acquired data requires an increased data budget;

- both those conditions demand for improved operating modes.

\section{The instrument}

The design of the HEPD-02 will have a number of improvements with minor changes with respect to the first design (identical box dimensions, mechanical interfaces and budgets).

The development of the tracker of HEPD-02 is ongoing, the new solution is based on 3 planes of ALPIDE CMOS pixel chips, developed for ALICE experiment at LHC [4]. Respect to HEPD01, this technology reduces systematic uncertainties on tracking giving a single-hit resolution up to 6 times better and do not suffer from multi-hit degeneracy.

The tracker is composed of five independent tracking modules $\left(225 \times 38 \times 12 \mathrm{~mm}^{3}\right.$, see Fig. 3), consisting each of three sensitive planes. The sensitive area of every layer is made up of 10 sensors, each containing $1024 \times 512$ square pixels of $28 \mu \mathrm{m}$. The control and read-out of the sensor is based on ultra-thin $(170 \mu \mathrm{m})$ flexible printed circuits on which the sensors are glued and electrically 


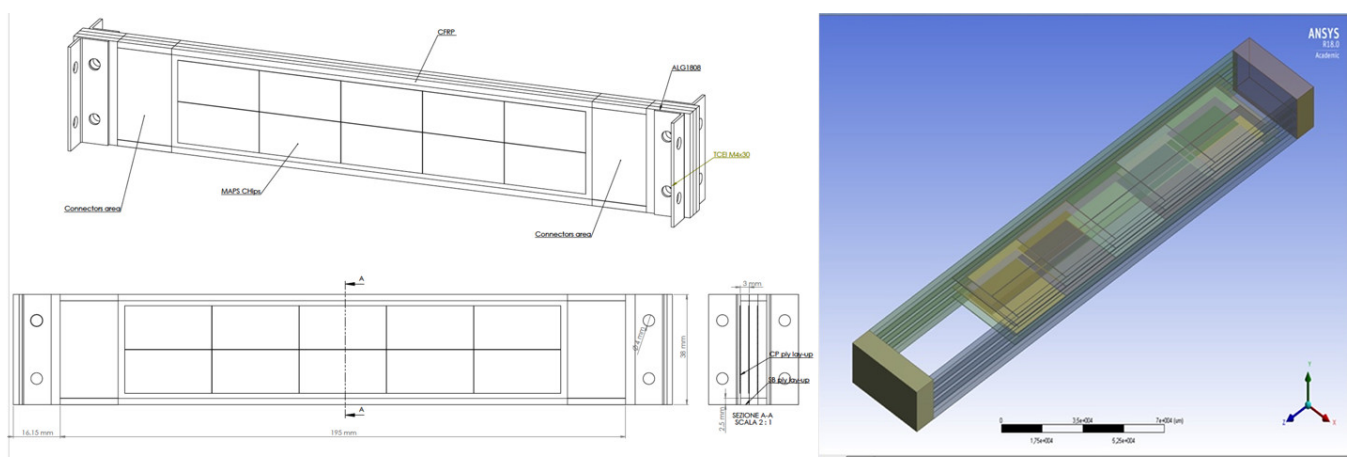

Figure 3: The layout of the tracking module of the tracker of HEPD-02.

connected. The modules are mechanically supported by a layer of reinforced carbon-fiber and an external frame.

To cope with the increased fluxes rates of polar orbits, a trigger system with two crossed layers of $3 \mathrm{~mm}$ thick scintillator planes read by light-guides has been designed (see Fig. 4). Each plane is segmented in 5 counters (dimensions $200 \times 30 \times 3 \mathrm{~mm}^{3}$ each excluding mechanics). Each counter is covered with reflective coating and read out by light-guides connected to PMTs (Hamamatsu R9880-210), one on each side. The signals coming from the segmented trigger layer, in coincidence with one or more signal coming from different planes of the calorimeter, provides the HEPD trigger signal. The new trigger will allow a fine-tuning of the trigger efficiency. Besides providing the trigger to the whole instrument, the trigger layer measures the loss of energy per unit length of each particle, improving the electron-proton discrimination. The $x-y$ segmentation also complements the tracking of the incoming particles and helps validating the tracking from the ALPIDE modules.

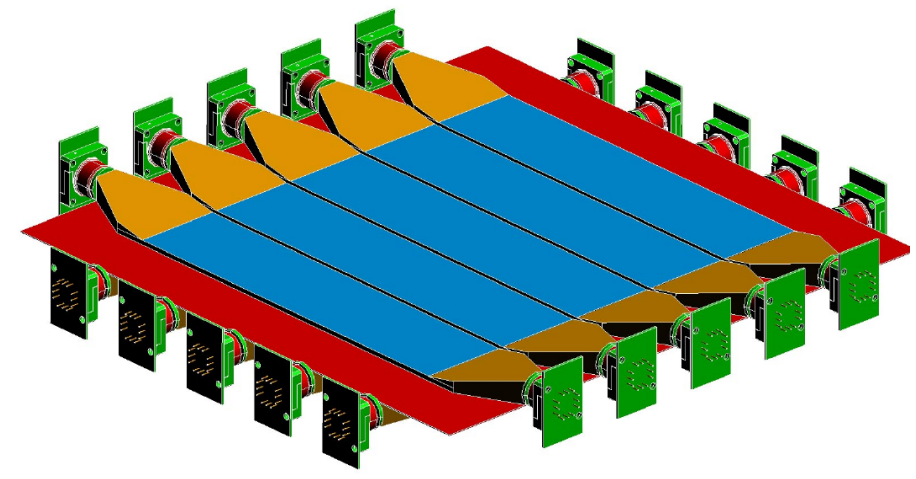

Figure 4: The layout of the trigger plane.

The calorimeter measures the energy of the incoming particles which are contained in it. It consists of 12 scintillator planes and of a $3 \times 3$ matrix of inorganic LYSO crystals on the bottom of the tower. Each plastic scintillator plane is covered with reflective coating and measures $150 \times 150 \times 10 \mathrm{~mm}^{3}$ (excluding mechanics). It is read out by two PMTs (Hamamatsu R9880-210) located on opposite corners.

The LYSO plane measures $150 \times 150 \times 40 \mathrm{~mm}^{3}$ and consists of a matrix of $3 \times 3$ blocks; each block is covered by reflective coating and read out by PMT (Hamamatsu R9880-210) located on 
the bottom face.

The veto consists of five plastic scintillator planes (four lateral and one at the bottom of the instrument), each covered with reflective coating and read out by two PMTs (Hamamatsu R9880210) located on opposite corners. The veto system allows to select particles whose signal is fully contained in the detector. The selection can be applied both during the acquisition and offline.

The HEPD-02 is contained in an aluminum box with a base plate linked to the satellite cabin space which allows to dissipate heat by contact. Two models of the instrument will be realized: qualification and flight model.

\subsection{The electronics}

Most of the improvements of HEPD-02 compared to HEPD-01 concern electronics. All the electronics of the HEPD-02 is designed with embedded "Hot/Cold" redundancy and all the components will be selected capable the industrial range $\left(-40^{\circ} \mathrm{C}\right.$ to $\left.+85^{\circ} \mathrm{C}\right)$. The trigger rate and the data stored have to be compliant with the maximum data transfer rate from the satellite, which is $50 \mathrm{~GB}$ per day.

The electronics system can be schematized into three blocks:

- tracker detector;

- scintillator detectors (trigger, energy and veto detectors);

- global control and data managing.

Each module has its own dedicated mechanics that allows anchoring to the HEPD-02 base plate and heat dissipation. Each detector block includes power chain for bias distribution and a data acquisition processing chain. The main power supply provides the low voltages to the detector electronics and the high bias voltages for PMTs. The communications between the various subsystems is carried out by means of the SPACEWIRE LITE protocol.

With regard to front-end electronics, the handling of the signals coming from the PMTs has been optimized. In addition, the board responsible for generating and distributing the system trigger, as well as for PMT signals processing and acquisition, has been redesigned. In the new board, in order to improve the management of PMT signals and the generation of triggers, the EASIROC ASIC has been substituted with the CITIROC chip, both by Weeroc.

The global control and data managing system has been updated: the previous architecture, based on an ADSP2189 and an ACTEL FPGA Flashpro3, will be replaced with a board based on Xilinx SoC. The design of the two high and low voltage power supply has also been reviewed and improved.

\section{Conclusions}

The HEPD-02 onboard CSES-02 satellite has been designed to improve the performances of the detector which is onboard the first satellite.

A new trigger system has been designed, along with a calorimeter and veto shielding upgrade. In addition, a new tracking system based on 3 planes of ALPIDE CMOS pixel chips, developed for ALICE experiment at LHC, will be used for the first time in space. 
The CSES-02 satellite is planned to be launched in less than 3 years and all the instruments on board will be full time operational.

\section{Acknowledgment}

This work was supported by the Italian Space Agency in the framework of the "Accordo Attuativo n. 2016-16-H0 Progetto Limadou Fase E/Scienza" (CUP F12F1600011005).

\section{References}

[1] G. Ambrosi et al., "The HEPD particle detector of the CSES satellite mission forinvestig ating seismo-associated perturbations of the Van Allen belts", Sci. China Technol. Sci., vol. 61, no. 5, pp. 643-652, May, 2018, DOI: https://doi.org/10.1007/s11431-018-9234-9

[2] P. Picozza et al., (CSES-Limadou collaboration), "Scientific goals and in-orbit performance of the High Energy Particle Detector on board the CSES satellite", ApJ Supplement, vol. 243, 2019

[3] V. Scotti, G. Osteria, for the CSES-Limadou Collaboration, "The electronics of the HEPD of the CSES experiment", Nuclear and Particle Physics Proceedings, 291-293: 118-121 https://doi.org/10.1016/j.nuclphysbps.2017.06.024

[4] R. Iuppa, "First use of CMOS Monolithic Active Pixel Sensors in space: the tracker of HEPD-02 based on the ALPIDE sensor", ICRC2019 